Proyecciones Journal of Mathematics

Vol. 28, No 3, pp. 253-270, December 2009.

Universidad Católica del Norte

Antofagasta - Chile

\title{
FUZZY PARA - LINDELOF SPACES
}

\author{
T. BAIJU \\ and \\ SUNIL JACOB JOHN \\ NATIONAL INSTITUTE OF TECHNOLOGY CALICUT, INDIA \\ Received : July 2009. Accepted : October 2009
}

\begin{abstract}
In this paper we introduce the concept of Para-Lindelof spaces in $L$-topological spaces by means of locally countable families of L-fuzzy sets. Further some characterizations of fuzzy para-Lindelofness and flintily para-Lindelofness in the weakly induced L-topological spaces are also obtained. More over the behavior of fuzzy para-Lindelof spaces under various types of maps such as fuzzy closed maps, fuzzy perfect maps are also investigated.
\end{abstract}

Keywords : L-Topology, Fuzzy para-Lindelofness, Flintily paraLindelofness, locally countable family.

2000 AMS Classification : $54 D 20,54 A 40$. 


\section{Introduction}

As a generalization of a set, the concept of fuzzy set was introduced by Zadeh [18]. Fuzzy topology comes as the generalization of general topology using the concept of a fuzzy set. In 1968 Chang [6] introduced the concept of fuzzy topology and Lowen [12] introduced a more natural definition of fuzzy topology.

Compactness and metrizability are the heart and soul of general topology. In 1944 J. Dieudonne [7] defined paracompactness as a natural generalization of compactness. Later several other covering properties such as meta-compactness, sub para-compactness, sub meta-compactness, paraLindelofness etc. have naturally evolved from para compactness. The concept of para-Lindelof spaces was introduced by J. Greever [9] in 1968 and further studies were conducted by Burke $([4,5])$, Fleissner-Reed [8].

The concept of paracompactness in fuzzy topology was introduced by Luo [13]. Authors have introduced the concept and studied some properties regarding metacompactness, subparacompactness, and submetacompactness in $L$-topological spaces in [14], [3], [2] respectively. In this paper we define locally countable families and introduce the concept of para-Lindelof spaces in $L$-topological spaces. Besides getting some characterization for para-Lindelof and flintily para-Lindelof in the weakly induced $L$-topological spaces, it is also seen that these properties are closed hereditary. Further the invariance of these properties under perfect maps is also proved.

Let $L$ be a complete lattice. Its universal bounds are denoted by $\perp$ and $T$. We presume that $L$ is consistent. i.e., $\perp$ is distinct from $T$. Thus $\perp \leq \alpha \leq \top$ for all $\alpha \in L$. We note $\vee \phi=\perp$ and $\wedge \phi=\top$. The two point lattice $\{\perp, \top\}$ is denoted by 2. A unary operation ' on $L$ is a quasicomplementation. It is an involution (ie., $\alpha^{\prime \prime}=\alpha$ for all $\alpha \in L$ ) that inverts the ordering. (ie., $\alpha \leq \beta$ implies $\left.\beta^{\prime} \leq \alpha^{\prime}\right)$. In $\left(L,^{\prime}\right)$ the DeMorgan laws hold: $(\vee A)^{\prime}=\wedge\left\{\alpha^{\prime}: \alpha \in A\right\}$ and $(\wedge A)^{\prime}=\vee\left\{\alpha^{\prime}: \alpha \in A\right\}$ for every $A \subset L$. Moreover, in particular, $\perp^{\prime}=\top$ and $\top^{\prime}=\perp$.

A molecule or co-prime element in a lattice $L$ is a join irreducible element in $L$ and the set of all non zero co-prime elements of $L$ is denoted by $M(L)$ and prime elements by $\operatorname{pr}(L)$. A complete lattice $L$ is completely distributive if it satisfies either of the logically equivalent CD1 or CD2 below: CD1: $\wedge_{i \in I}\left(\vee_{j \in J_{i}} a_{i, j}\right)=\vee_{\phi \in \Pi J_{i} i \in I}\left(\wedge_{i \in I} a_{i, \phi_{(i)}}\right)$

CD2: $\vee_{i \in I}\left(\wedge_{j \in J_{i}} a_{i, j}\right)=\wedge_{\phi \in \Pi J_{i} i \in I}\left(\vee_{i \in I} a_{i, \phi_{(i)}}\right)$

for all $\left\{\left\{a_{i j}: j \in J_{i}\right\}: i \in I\right\} \subset P(L) \backslash\{\phi\}$,

If $L$ is a complete lattice, then for a set $X, L^{X}$ is the complete lattice of 
all maps from $X$ into $L$, called $L$-sets or $L$-subsets of $X$. Under point-wise ordering, $a \leq b$ in $L^{X}$ if and only if $a(x) \leq b(x)$ in $L$ for all $x \in X$. If $A \subset X, 1_{A} \in 2^{X} \subset L^{X}$ is the characteristic function of $A$. The constant member of $L^{X}$ with value $\alpha$ is denoted by $\alpha$ itself. Usually we will not distinguish between a crisp set and its characteristic function. Wang [15] proved that a complete lattice is completely distributive if and only if for each $\alpha \in L$, there exists $B \subseteq L$ such that (i) $a=\vee A$ and (ii) if $A \subseteq L$ and $a \leq \vee B$, then for each $b \in B$, there exists $c \in A$ such that $b \leq c$. $B$ is called the minimal set of $a$ and $\beta(a)$ denote the union of all minimal sets of $a$. Again $\beta^{*}(a)=\beta(a) \cap M(L)$. Clearly $\beta(a)$ and $\beta^{*}(a)$ are minimal sets of $a$.

For $\alpha \in L$ and $A \in L^{X}$, we use the following notations.

$A_{[\alpha]}=\{x \in X: A(x) \geq \alpha\}$

$A^{[\alpha]}=\{x \in X: A(x) \leq \alpha\}$

$A^{(\alpha)}=\{x \in X: A(x) \nsupseteq \alpha\} ;$

$A_{(\alpha)}=\{x \in X: A(x) \not \leq \alpha\}$.

Clearly $L^{X}$ has a quasi complementation ' defined point-wisely $\alpha^{\prime}(x)=$ $\alpha(x)^{\prime}$ for all $\alpha \in L$ and $x \in X$. Thus the DeMorgan laws are inherited by $\left(L^{X},{ }^{\prime}\right)$.

Let $\left(L,^{\prime}\right)$ be a complete lattice equipped with an order reversing involution and $X$ be any non empty set. A subfamily $\tau \subset L^{X}$ which is closed under the formation of sups and finite infs (both formed in $L^{X}$ ) is called an $L$-topology on $X$ and its members are called open $L$-sets. The pair $(X, \tau)$ is called an $L$-topological space $(L$-ts). The category of all $L$-topological spaces, together with $L$-continuous mappings and the composition and identities of set is denoted by $L$-Top. Quasi complements of open $L$-sets are called closed $L$-sets.

We know that the set of all non zero co-prime elements in a completely distributive lattice is $\vee$-generating. Moreover for a continuous lattice $L$ and a topological space $(X, T), T=i_{L} \omega_{L}(T)$ is not true in general. By proposition 3.5 in Kubiak [11] we know that one sufficient condition for $T=i_{L} \omega_{L}(T)$ is that $L$ is completely distributive.

In [16] Wang extended the Lowen functor $\omega$ for completely distributive lattices as follows: For a topological space $(X, T),(X, \omega(T))$ is called the induced space of $(X, T)$ where $\omega(T)=\left\{A \in L^{X}: \forall \alpha \in M(L), A^{\left(\alpha^{\prime}\right)} \in T\right\}$. In 1992 Kubiak also extended the Lowen functor $\omega_{L}$ for a complete lattice $L$. In fact when $L$ is completely distributive, $\omega_{L}=\omega$.

An $L$-topological space $(X, \tau)$ is called weakly induced space if $\forall \alpha \in$ $M(L), \forall A \in \tau$ it is true that $A^{\left(\alpha^{\prime}\right)} \in[\tau]$ where $[\tau]$ is the set of all crisp 
open sets in $\tau$.

Based on these facts, in this paper we use a complete, completely distributive lattice $L$ in $L^{X}$. For a standardized basic fixed-basis terminology, we follow Hohle and Rodabaugh [10].

\section{Preliminaries and Basic Definitions}

\subsection{Definition}

[17] Let $(X, \tau)$ be an $L$-ts. A fuzzy point $x_{\alpha}$ is quasi coincident with $D \in L^{X}$ (and write $\left.x_{\alpha} \prec D\right)$ if $x_{\alpha} \not \leq D^{\prime}$. Also $D$ quasi coincides with $E$ at $x$ ( $D q$ $E$ at $x)$ if $D(x) \not \leq E^{\prime}(x)$. We say $D$ quasi coincident with $E$ and write $D$ $q E$ if $D q E$ at $x$ for some $x \in X$. Further $D \neg q E$ means $D$ not quasi coincides with $E$. We say $U \in \tau$ is quasi coincident $n b d$ of $x_{\alpha}(Q-n b d)$ if $x_{\alpha} \prec U$. The family of all $Q$-nbds of $x_{\alpha}$ is denoted by $Q_{\tau}\left(x_{\alpha}\right)$ or $Q\left(x_{\alpha}\right)$.

\subsection{Definition}

[17] Let $(X, \tau)$ be an $L$-ts, $A \in L^{X} . \Phi \subset L^{X}$ is called a $Q$-cover of $A$ if for every $x \in \operatorname{Supp}(A)$, there exist $U \in \Phi$ such that $x_{A(x)} \prec U$. $\Phi$ is a $Q$-cover of $(X, \tau)$ if $\Phi$ is a $Q$ cover of $\top$. If $\alpha \in M(L)$, then $\mathbf{C} \in \tau$ is an $\alpha-Q-n b d$ of $A$ if $\mathbf{C} \in Q\left(x_{\alpha}\right)$ for every $x_{\alpha} \leq A$. $\Phi$ is called an $\alpha$-Q-cover of $A$, if for each $x_{\alpha} \leq A$, there exists $U \in \Phi$ such that $x_{\alpha} \prec U$. $\Phi$ is called an open $\alpha$ - $Q$-cover of $A$ if $\Phi \subset \tau$ and $\Phi$ is an $\alpha$ - $Q$-cover of $A$. $\Phi_{0} \subset L^{X}$ is called a sub $\alpha$ - $Q$-cover of $A$ if $\Phi_{0} \subset \Phi$ and $\Phi_{0}$ is also an $\alpha$ - $Q$-cover of $A$. $\Phi$ is called an $\alpha^{-}-Q$ cover of $A$, if there exists $\gamma \in \beta^{*}(\alpha)$ such that $\Phi$ is $\gamma$-Q-cover of $A$.

\subsection{Definition}

[17] Let $(X, \tau)$ be an $L$-ts, $D \in L^{X}$. $D$ is called $N$-compact if for every $\alpha \in M(L)$, every open $\alpha-Q$ cover of $D$ has a finite sub family which is an $\alpha^{-}-Q$ cover of $D .(X, \tau)$ is called $N$-compact if $\top$ is $N$-compact.

\subsection{Definition}

[?] Let $(X, \tau)$ be an $L$-ts, $\mathbf{A}=\left\{A_{t}: t \in T\right\} \subseteq L^{X}, x_{\lambda} \in M\left(L^{X}\right)$. $\mathbf{A}$ is called locally finite at $x_{\lambda}$, if there exist $U \in Q\left(x_{\lambda}\right)$ and a finite subset $T_{0}$ of $T$ such that $t \in T \backslash T_{0} \Rightarrow A_{t} \neg q U$. And $\mathbf{A}$ is called *-locally finite at $x_{\lambda}$ if there exist $U \in Q\left(x_{\lambda}\right)$ and a finite subset $T_{0}$ of $T$ such that 
$t \in T_{0} \Rightarrow \chi_{\left(A_{t}\right)_{(\perp)}} \neg q U$. A is called locally finite (*-locally finite) for short, if $\mathbf{A}$ is locally finite(*-locally finite) at every molecule $x_{\lambda} \in M\left(L^{X}\right)$.

\subsection{Definition}

[14] Let $(X, \tau)$ be an $L$-ts. $\mathbf{A}=\left\{A_{t}: t \in T\right\} \subset L^{X}, x_{\lambda} \in M\left(L^{X}\right) . \mathbf{A}$ is called point finite at $x_{\lambda}$ if $x_{\lambda} \prec A_{t}$ for at most finitely many $t \in T$. And $\mathbf{A}$ is ${ }^{*}$-point finite at $x_{\lambda}$ if there exists at most finitely many $t \in T$ such that $x_{\lambda} \prec \chi_{\left(A_{t}\right)_{(\perp)}}$. A is called point finite (resp. ${ }^{*}$-point finite) for short, if $\mathbf{A}$ is point finite (resp. ${ }^{*}$-point finite) at every molecule $x_{\lambda}$ of $L^{X}$.

\subsection{Definition}

Let $(X, \tau)$ be an $L$-ts, $\mathbf{A}=\left\{A_{t}: t \in T\right\} \subseteq L^{X}, x_{\lambda} \in M\left(L^{X}\right)$. $\mathbf{A}$ is called locally countable at $x_{\lambda}$, if there exist $U \in Q\left(x_{\lambda}\right)$ and a countable subset $T_{0}$ of $T$ such that $t \in T \backslash T_{0} \Rightarrow A_{t} \neg q U$. And $\mathbf{A}$ is called *-locally countable at $x_{\lambda}$ if there exist $U \in Q\left(x_{\lambda}\right)$ and a countable subset $T_{0}$ of $T$ such that $t \in T_{0} \Rightarrow \chi_{\left(A_{t}\right)_{(\perp)}} \neg q U$. A is called locally countable (*-locally countable) for short, if $\mathbf{A}$ is locally countable (*-locally countable) at every molecule $x_{\lambda} \in M\left(L^{X}\right)$.

The previous notions "locally countable family" is defined for $L$-ts. They can be also defined for $L$-subsets:

\subsection{Definition}

Let $(X, \tau)$ be an $L$-ts. $A \in L^{X}, \mathbf{A}=\left\{A_{t}: t \in T\right\} \subset L^{X}, x_{\lambda} \in M\left(L^{X}\right)$. $\mathbf{A}$ is called locally countable in $A$, if $\mathbf{A}$ is locally countable at every molecule $x_{\lambda} \in M(\downarrow A)$.

\subsection{Definition}

Let $(X, \tau)$ be an $L$-ts. $\mathbf{A}=\left\{A_{t}: t \in T\right\} \subseteq L^{X}, B \in L^{X}$.

$\mathbf{A}$ is called $\sigma$-locally countable in $B$ if $\mathbf{A}$ is the countable union of sub families which are locally countable in $B$. A is called $\sigma$-locally countable for short, if $\mathbf{A}$ is $\sigma$-locally countable in $\top$.

\subsection{Definition}

[17] Let $(X, \tau)$ be an $L$-ts. Then by $[\tau]$ we denote the family of support sets of all crisp subsets in $\tau .(X,[\tau])$ is a topology and it is the background 
space. $(X, \tau)$ is weakly induced if $U \in \tau$ is a lower semi continuous function from the background space $(X,[\tau])$ to $L$.

\subsection{Definition}

[17] Let $(X, \tau)$ be an $L$-ts. $(X, \tau)$ is called weakly $\alpha$-induced if $U_{(\alpha)} \in[\tau]$ for every $U \in \tau$.

\subsection{Proposition}

[17] Let $(X, \tau)$ be an $L$-ts. Then the following conditions are equivalent.

(i) $(X, \tau)$ is weakly induced.

(ii) $(X, \tau)$ is weakly $\gamma$-induced for every $\gamma \in \operatorname{pr}(L)$.

(iii) $(X, \tau)$ is weakly $\alpha$-induced for every $\alpha \in L$.

\subsection{Definition}

[17] For a property $P$ of ordinary topological space, a property $P^{*}$ of $L$-ts is called a good $L$-extension of $P$, if for every ordinary topological space $(X, T),(X, T)$ has the property $P$ if and only if $\left(X, \omega_{L}(T)\right)$ has property $P^{*}$. In particular when $L=[0,1]$ we say $P^{*}$ is a good extension of $P$. Where $\omega_{L}(T)$ is the family of all lower semi continuous function from $(X, T)$ to $L$.

\subsection{Definition}

[17] A collection $\mathbf{A}$ refines a collection $\mathbf{B}(\mathbf{A}<\mathbf{B})$ if for every $A \in \mathbf{A}$, there exists $B \in \mathbf{B}$ such that $A \leq B$.

\subsection{Definition}

[17] Let $(X, \tau)$ be an $L$-ts. $\mathbf{A}=\left\{A_{t}: t \in T\right\} \subseteq L^{X}$ is a closure preserving collection if for every subfamily $\mathbf{A}_{0}$ of $\mathbf{A}, c l\left[\vee \mathbf{A}_{0}\right]=\vee\left[c l \mathbf{A}_{0}\right]$.

\subsection{Proposition}

[17] Let $(X, \tau)$ be an $L$-ts. $\mathbf{A} \subset L^{X}$ is closure preserving. Then for every sub family $\mathbf{A}_{0}=\left\{A_{t}: t \in T\right\} \subset \mathbf{A}, \vee_{t \in T} c l A_{t}$ is a closed subset.

\subsection{Theorem}

Every locally countable family of subsets is closure preserving. 
Proof. Let $\mathbf{A} \subset L^{X}$ is locally countable, $\mathbf{A}_{0}=\left\{A_{t}: t \in T\right\} \subset \mathbf{A}$, then $\mathbf{A}_{0}$ is locally countable. Since $\vee\left(\operatorname{cl} \mathbf{A}_{0}\right) \leq \operatorname{cl}\left(\vee \mathbf{A}_{0}\right)$ is clear it is sufficient to prove that $c l\left(\vee \mathbf{A}_{0}\right) \leq \vee\left(c l \mathbf{A}_{0}\right)$. Suppose $x_{\alpha} \in M\left(\downarrow c l\left(\vee \mathbf{A}_{0}\right)\right)$. Since $\mathbf{A}_{0}$ is locally countable, there exist $U \in Q\left(x_{\alpha}\right)$ such that $\Rightarrow A_{t} \neg q U$ for every $t \in T \backslash T_{0}$ where $T_{0}$ is a countable subset of $T$. This implies that $A_{t} \leq U^{\prime}$ for every $t \in T \backslash T_{0}$. If $x_{\alpha} \not \leq \vee\left(\mathrm{cl} \mathbf{A}_{0}\right)$, then $x_{\alpha} \not \leq c l \mathbf{A}_{t}$ for every $t \in T_{0}$ and hence there exist $U_{t} \in Q\left(x_{\alpha}\right)$ such that $A_{t} \leq U_{t}^{\prime}$. Since $T_{0}$ is countable, $V=U \wedge\left(\vee_{t \in T_{0}} U_{t}\right) \in Q\left(x_{\alpha}\right)$ and $A_{t} \leq V^{\prime}$ for every $t \in T$. So $\vee_{t \in T} A_{t} \leq V^{\prime}$ and hence $x_{\alpha} \leq \operatorname{cl}\left(\vee_{\mathbf{A}_{0}}\right)=\operatorname{cl}\left(\vee_{t \in T} A_{t}\right) \leq \operatorname{cl}\left(V^{\prime}\right)=V^{\prime}$. That is $x_{\alpha}$ is not quasi coincidence with $V$, which is a contradiction that $V \in Q\left(x_{\alpha}\right)$. Therefore $x_{\alpha} \in \vee\left(\operatorname{cl} \mathbf{A}_{0}\right)$ and thus $c l\left(\vee \mathbf{A}_{0}\right)=\vee\left(c l \mathbf{A}_{0}\right)$.

\subsection{Definition}

[14] A collection $\mathbf{U}$ of fuzzy subsets of an $L$-topological space $(X, \tau)$ is said to be well monotone if the subset relation ' $<$ ' is a well order on $\mathbf{U}$.

\subsection{Definition}

[14] A collection $\mathbf{U}$ of fuzzy subsets of an $L$-topological space $(X, \tau)$ is said to be directed if $U, V \in \mathbf{U}$ implies there exists $W \in \mathbf{U}$ such that $U \vee V<W$.

\subsection{Definition}

Let $(X, \tau)$ be an $L$-ts, $A \in L^{X}, \mathbf{B} \subset L^{X}$. Then $\operatorname{st}(A, \mathbf{B})=\vee\{B \in$ $\mathbf{B}: B q A\}$ is defined as the star of $\mathbf{B}$ about $A$. If $x_{\lambda} \in M\left(L^{X}\right)$, then $\operatorname{st}\left(\left\{x_{\lambda}\right\}, \mathbf{B}\right)$ is denoted by $\operatorname{st}\left(x_{\lambda}, \mathbf{B}\right)$.

\subsection{Definition}

Let $(X, \tau)$ be an $L$-ts. $\mathbf{A}=\left\{A_{t}: t \in T\right\} \subseteq L^{X}$ is a interior preserving collection if for every subfamily $\mathbf{A}_{0}$ of $\mathbf{A}$, int $\left[\wedge \mathbf{A}_{0}\right]=\wedge\left[\right.$ int $\left.\mathbf{A}_{0}\right]$.

\section{Para-Lindelof Spaces}

\subsection{Definition}

[17] Let $(X, \tau)$ be an $L$-ts, $A \in L^{X}, \alpha \in M(L) . \quad A$ is called $\alpha$-Lindelof if every open $\alpha$ - $Q$-cover of $A$ has a countable subfamily which is also an $\alpha$ - $Q$-cover of $A$. $A$ is Lindelof if $A$ is $\alpha$ - Lindelof for every $\alpha \in M(L)$. And $(X, \tau)$ is Lindelof if $\top$ is Lindelof. 


\subsection{Definition}

Let $(X, \tau)$ be an $L$-ts, $A \in L^{X}, \alpha \in M(L) . A$ is called $\alpha$-para-Lindelof ( $\alpha^{*}$-para-Lindelof) if for every open $\alpha$ - $Q$-cover $\Phi$ of $A$, there exist an open refinement $\Psi$ of $\Phi$ which is locally countable (*-locally countable) in $A$ and $\Psi$ is also an $\alpha$ - $Q$-cover of $A$. $A$ is para-Lindelof (*-para-Lindelof) if $A$ is $\alpha$-para-Lindelof ( $\alpha^{*}$-para-Lindelof) for every $\alpha \in M(L) .(X, \tau)$ is para-Lindelof (*-para-Lindelof) if $T$ is para-Lindelof (*-para-Lindelof).

\subsection{Definition}

Let $(X, \tau)$ be an $L$-ts, $\alpha \in M(L) .(X, \tau)$ is called $\sigma$-para-Lindelof if for every open $\alpha$ - $Q$-cover $\Phi$ of $X$, there exist an open refinement $\Psi$ of $\Phi$ which is $\sigma$-locally countable in $X$ and also an $\alpha$-Q-cover of $X$.

\subsection{Proposition}

Let $(X, \tau)$ be an $L$-ts, $A \in L^{X}, \alpha \in M(L)$. Then

(i) $A$ is $\alpha^{*}$-para-Lindelof $\Rightarrow A$ is $\alpha$-para-Lindelof.

(ii) $A$ is $*$-para-Lindelof $\Rightarrow A$ is para-Lindelof.

Para-Lindelof and ${ }^{*}$-Para-Lindelof are hereditary with respect to closed subsets.

\subsection{Theorem}

Let $(X, \tau)$ be an $L$-ts, $A \in L^{X}, \alpha \in M(L), B \in \tau^{\prime}$. Then

(i) $A$ is $\alpha$-para-Lindelof $\Rightarrow A \wedge B$ is $\alpha$-para-Lindelof.

(ii) $A$ is para-Lindelof $\Rightarrow A \wedge B$ is para-Lindelof.

Proof. We need to prove only (i). Suppose that $\mathbf{U}$ is an open $\alpha-Q$ cover of $A \wedge B$. Take $\mathbf{V}=\mathbf{U} \cup\left\{B^{\prime}\right\}$. Now clearly $\mathbf{V}$ is an open $\alpha$ - $Q$ cover of $A$. Since $A$ is $\alpha$-para-Lindelof, $\mathbf{V}$ has an open refinement $\mathbf{W}$ such that $\mathbf{W}$ is locally countable in $A$ and is also an $\alpha$-Q-cover of $A$. Take $\mathbf{W}_{0}=\{W \in \mathbf{W}: \exists U \in \mathbf{U}, W \leq U\}$. Now we show that $\mathbf{W}_{0}$ is the required locally countable refinement of $\mathbf{V}$ which is also an $\alpha$ - $Q$-cover of $A \wedge B$. Clearly $\mathbf{W}_{0}$ is a locally countable refinement. Let $x_{\alpha} \leq A \wedge B \leq A$, since $\mathbf{W}$ is an $\alpha$ - $Q$-cover of $A$, there exist $W \in \mathbf{W}$ such that $x_{\alpha} \prec W$. Since $x_{\alpha} \leq B, B \not B^{\prime}$, i.e. $W \not \leq B^{\prime}$. Since $\mathbf{W}$ is a refinement of $\mathbf{V}=\mathbf{U} \cup\left\{B^{\prime}\right\}$, $\exists U \in \mathbf{U}$ such that $W \leq U$. Thus $W \in \mathbf{W}_{0}$ and hence $x_{\alpha} \prec W \in \mathbf{W}_{0}$.

A similar theorem holds for $\alpha^{*}$-para-Lindelof and ${ }^{*}$-para-Lindelof spaces also. 


\subsection{Theorem}

Let $(X, \tau)$ be an $L$-ts, $A \in L^{X}, \alpha \in M(L), B \in \tau^{\prime}$. Then

(i) $A$ is $\alpha^{*}$-para-Lindelof $\Rightarrow A \wedge B$ is $\alpha^{*}$-para-Lindelof.

(ii) $A$ is ${ }^{*}$-para-Lindelof $\Rightarrow A \wedge B$ is ${ }^{*}$-para-Lindelof.

\subsection{Theorem}

Let $(X, \tau)$ be a weakly induced $L$-ts. Then the following conditions are equivalent

(i) $(X, \tau)$ is para-Lindelof;

(ii) There exist $\alpha \in M(L)$ such that $(X, \tau)$ is $\alpha$-para-Lindelof;

(iii) $(X,[\tau])$ is para-Lindelof.

Proof. $\quad$ (i) $\Rightarrow$ (ii) is obvious.

(ii) $\Rightarrow$ (iii): Let $\mathbf{U} \subset[\tau]$ be an open cover of $X$. Now $\mathbf{U}^{*}=\left\{\chi_{U}: U \in \mathbf{U}\right\}$ is an open $\alpha$ - $Q$-cover of $T$ and it has a locally countable refinement $\mathbf{V}$ which is also an $\alpha$-Q-cover of $T$.

Let $\mathbf{W}=\left\{V_{\left(\alpha^{\prime}\right)}: V \in \mathbf{V}\right\}$. Clearly $\mathbf{W}$ is both a refinement of $\mathbf{U}$ and a cover of $X$. Since $(X, \tau)$ is weakly induced, we have $\mathbf{W} \subset[\tau]$. Now we want to prove that $\mathbf{W}$ is locally countable. Let $x \in X$. Since $(X, \tau)$ is $\alpha$-para-Lindelof, there exist $B \in Q\left(x_{\alpha}\right)$ such that $B$ only quasi coincides with a countable number of members $V_{0}, V_{1}, V_{2}, \cdots$ of $\mathbf{V}$. Let $O=B_{(\perp)}$. By the weakly induced property of $(X, \tau), O \in[\tau]$. For every $V \in \mathbf{V}$, if $O \cap V_{\left(\alpha^{\prime}\right)} \neq \phi$, then there exist an ordinary point $y \in O \cap V_{\left(\alpha^{\prime}\right)}$, and hence $B(y) \not \leq \perp, V(y) \not \leq \alpha^{\prime}$. Therefore $V(y)^{\prime}<\alpha$ and it follows that $B(y) \not Z V(y)^{\prime}$ and thus $B q V$. So $V \in\left\{V_{0}, V_{1}, V_{2}, \cdots\right\}$ and $O$ intersects only a countable number of members $V_{0\left(\alpha^{\prime}\right)}, V_{1\left(\alpha^{\prime}\right)}, V_{2\left(\alpha^{\prime}\right)}, \cdots$ of $\mathbf{W}$. Hence $(X,[\tau])$ is para-Lindelof.

(iii) $\Rightarrow$ (i): Suppose that $\alpha \in M(L)$ and $\mathbf{U} \subset \tau$ be an open $\alpha$ - $Q$-cover of $\top$. Since $(X, \tau)$ is weakly induced $\mathbf{U}^{*}=\left\{U_{\left(\alpha^{\prime}\right)}: U \in \mathbf{U}\right\}$ is an open cover of $(X,[\tau])$. Since $(X,[\tau])$ is para-Lindelof, there exist a refinement $\mathbf{V}$ of $\mathbf{U}^{*}$ which is also a locally countable cover of $X$. For every $V \in \mathbf{V}$, let $U_{V} \in \mathbf{U}$ such that $V \subset U_{V\left(\alpha^{\prime}\right)}$. Let $\mathbf{W}=\left\{\chi_{V} \wedge U_{V}: V \in \mathbf{V}\right\}$. Now clearly $\mathbf{W}$ is both a refinement of $\mathbf{U}$ and an $\alpha$-Q-cover of $T$. Now we will prove that $\mathbf{W}$ is locally countable. Let $x_{\alpha} \in M\left(L^{X}\right)$. Then since $\mathbf{V}$ is locally countable, there exist a neighbourhood $B$ of $x$ such that $B$ intersects with $V_{i}$ for countably many $V_{i} \in \mathbf{V}$. Now we have $\chi_{B} \in Q\left(x_{\alpha}\right)$. We will show that $\chi_{B} q \chi_{V_{i}} \wedge U_{V_{i}}$ for at most countably many $i$. For if possible $\chi_{B} q \chi_{V} \wedge U_{V}$ for uncountably many $V \in \mathbf{V}$. Then $\chi_{B} q \chi_{V}$ or $\chi_{B}$ 
$q U_{V}$ for uncountably many $V \in \mathbf{V}$. In both cases $B$ intersects with $V$ for uncountably many $V \in \mathbf{V}$, which is a contradiction and hence $W$ is locally countable. Therefore $(X, \tau)$ is $\alpha$-para-Lindelof. This completes the proof.

\subsection{Theorem}

Let $(X, \tau)$ be a weakly induced $L$-ts. Then the following conditions are equivalent

(i) $(X, \tau)$ is *-para-Lindelof;

(ii) There exist $\alpha \in M(L)$ such that $(X, \tau)$ is $\alpha^{*}$-para-Lindelof;

(iii) $(X,[\tau])$ is para-Lindelof.

Proof. $\quad$ (i) $\Rightarrow$ (ii) is obvious.

(ii) $\Rightarrow$ (iii): Let $\mathbf{U} \subset[\tau]$ be an open cover of $X$. Now $\mathbf{U}^{*}=\left\{\chi_{U}: U \in \mathbf{U}\right\}$ is an open $\alpha$ - $Q$-cover of $\top$ and it has a locally countable refinement $\mathbf{V}$ which is also an $\alpha$ - $Q$-cover of $\top$.

Take $\mathbf{W}=\left\{V_{\left(\alpha^{\prime}\right)}: V \in \mathbf{V}\right\}$ then $\mathbf{W}$ is both a refinement of $\mathbf{U}$ and a cover of $X$. Since $(X, \tau)$ is weakly induced, we have $\mathbf{W} \subset[\tau]$. Now we want to prove that $\mathbf{W}$ is locally countable. Let $x \in X$. Since $(X, \tau)$ is $\alpha^{*}$-para-Lindelof, there exist $B \in Q\left(x_{\alpha}\right)$ such that $\chi_{B(\perp)}$ only quasi coincides with a countable number of members $V_{0}, V_{1}, V_{2}, \cdots$ of $\mathbf{V}$. Then $x \in B_{(\perp)}$. By the weakly induced property of $(X, \tau), B_{[\perp]} \in[\tau]$, so $B_{(\perp)}$ is a neighbourhood of $x$. For every $V \in \mathbf{V}$, if $B_{(\perp)} \cap V_{\left(\alpha^{\prime}\right)} \neq \phi$, then there exist an ordinary point $y \in B_{(\perp)} \cap V_{\left(\alpha^{\prime}\right)}, V(y) \not \leq \alpha^{\prime}, V(y)>\perp, V(y)^{\prime}<\perp$. So $\chi_{B(\perp)}(y)=\top \not \leq V(y)^{\prime}, \chi_{B(\perp)} q V, V \in\left\{V_{0}, V_{1}, V_{2}, \cdots\right\}$. Therefore the neighbourhood $B_{(\perp)}$ is of $x$ intersects a countable number of members $V_{0\left(\alpha^{\prime}\right)}, V_{1\left(\alpha^{\prime}\right)}, V_{2\left(\alpha^{\prime}\right)}, \cdots$ of $\mathbf{W}$, thus $\mathbf{W}$ is locally countable in $X$. Hence $(X,[\tau])$ is para-Lindelof.

(iii) $\Rightarrow$ (i): Suppose that $\alpha \in M(L)$ and $\mathbf{U} \subset \tau$ be an open $\alpha$ - $Q$-cover of $\top$. Since $(X, \tau)$ is weakly induced $\mathbf{U}^{*}=\left\{U_{\left(\alpha^{\prime}\right)}: U \in \mathbf{U}\right\}$ is an open cover of $(X,[\tau])$. Since $(X,[\tau])$ is para-Lindelof, there exist a locally countable and open refinement $\mathbf{V}$ of $\mathbf{U}^{*}$ which is also a cover of $X$. For every $V \in \mathbf{V}$, let $U_{V} \in \mathbf{U}$ such that $V \subset U_{V\left(\alpha^{\prime}\right)}$. Let $\mathbf{W}=\left\{\chi_{V} \wedge U_{V}: V \in \mathbf{V}\right\}$. Then $\mathbf{W} \subset \tau$ is clearly a refinement of $\mathbf{U}$ and an $\alpha$-Q-cover of $\top$. Now we will prove that $\mathbf{W}$ is *-locally countable. Let $x_{\alpha} \in M\left(L^{X}\right)$ and $B \in Q\left(x_{\alpha}\right)$. If possible let $\chi\left(\chi_{V} \wedge U_{V}\right)_{(\perp)} q B$ for uncountably many $V \in \mathbf{V}$. That is $\chi_{V} \wedge \chi_{U V(\perp)} q B$ for uncountably many $V \in \mathbf{V}$. And hence $\chi_{V} q B$ or $\chi_{U V(\perp)} q B$ for uncountably many $V \in \mathbf{V}$. In both cases $V$ intersects with the neighbourhood of $x$ for uncountably many $V \in \mathbf{V}$ which is a 
contradiction that $\mathbf{V}$ is locally countable. Hence $W$ is *-locally countable and this completes the proof.

\subsection{Theorem}

Let $(X, \tau)$ be an $L$-ts. Then the following are equivalent

(i) $(X, \tau)$ is para-Lindelof;

(ii) For every open $\alpha$-Q-cover $\mathbf{A}$ of $(X, \tau)$, there is a locally countable refinement $\mathbf{B}$ such that if $x_{\alpha} \in M\left(L^{X}\right)$ then $x_{\alpha} \in \operatorname{int}\left(\operatorname{st}\left(x_{\alpha}, \mathbf{B}\right)\right)$.

Proof. $\quad($ i $) \Rightarrow$ (ii) is obvious.

(ii) $\Rightarrow$ (i): Suppose $\mathbf{A}=\left\{A_{t}: t \in T\right\}$ is an open $\alpha$ - $Q$-cover of $T$. Let $\mathbf{B}=\left\{B_{t}: t \in T\right\}$ be a locally countable refinement as given in (ii). Let $\mathbf{C}$ be an open $\alpha$ - $Q$-cover of $\top$ such that every element of $\mathbf{C}$ intersects at most countably many elements of $\mathbf{B}$. Then for every $x_{\alpha} \in M\left(L^{X}\right)$, there is a locally countable refinement $\mathbf{D}$ of $\mathbf{C}$ such that $x_{\alpha} \in \operatorname{int}\left(\operatorname{st}\left(x_{\alpha}, \mathbf{D}\right)\right)$.

For each $B \in \mathbf{B}$, take $A_{B} \in \mathbf{A}$ such that $B \leq A_{B}$ and let $G_{B}=$ $\operatorname{int}(\operatorname{st}(B, \mathbf{D})) \wedge A_{B}$. Then clearly $\mathbf{G}=\left\{G_{B}: B \in \mathbf{B}\right\}$ is an $\alpha$-Q-cover of $\top$ and hence is an open refinement of $\mathbf{A}$. To show $\mathbf{G}$ is locally countable, let $x_{\alpha} \in M\left(L^{X}\right)$ and $W \in Q\left(x_{\alpha}\right)$ such that $W$ intersects only countably many elements of $\mathbf{D}$. Now since each $D \in \mathbf{D}$ intersects only countably many elements of $\mathbf{B}$, it follows that $W$ intersects only countably many elements of $\{\operatorname{st}(B, \mathbf{D}): B \in \mathbf{B}\}$. Hence $\mathbf{G}$ is locally countable and the theorem is proved.

Similar to Theorem 3.9 we can prove the following result:

\subsection{Theorem}

Let $(X, \tau)$ be an $L$-ts. Then the following are equivalent

(i) $(X, \tau)$ is $\sigma$-para-Lindelof;

(ii) For any open $\alpha$-Q-cover $\mathbf{A}$ of $(X, \tau)$, there is a $\sigma$-locally countable refinement $\mathbf{B}=\cup \mathbf{B}_{i}$ such that if $x_{\alpha} \in M\left(L^{X}\right)$ then $x_{\alpha} \in \operatorname{int}\left(\operatorname{st}\left(x_{\alpha}, \mathbf{B}_{k}\right)\right)$ for some $k \in \mathbf{N}$.

\section{Flintily Para-Lindelof Spaces}

\subsection{Definition}

Let $(X, \tau)$ be an $L$-ts. $A \in L^{X}, \mathbf{A}=\left\{A_{t}: t \in T\right\} \subseteq L^{X}, x_{\lambda} \in M\left(L^{X}\right)$. A is called flintily locally countable at $x_{\lambda}$ if there exist $U \in Q\left(x_{\lambda}\right) \cap \operatorname{crs}(\tau)$ 
and a countable subset $T_{0}$ of $T$ such that $t \in T \backslash T_{0} \Rightarrow A_{t} \neg q U$. And $\mathbf{A}$ is called flintily locally countable in $A$, if $\mathbf{A}$ is flintily locally countable at every molecule $x_{\lambda} \in M(\downarrow A)$. $\mathbf{A}$ is called flintily locally countable for short, if $\mathbf{A}$ is flintily locally countable in $T$.

\subsection{Theorem}

In $L$-ts the following implications hold

Flintily local countable $\Rightarrow{ }^{*}$-local countable $\Rightarrow$ local countable

\subsection{Proposition}

Let $(X, \tau)$ be an $L$-ts, $\left\{A_{t}: t \in T\right\} \subseteq L^{X}, x_{\lambda} \in M\left(L^{X}\right)$. Then

(i) $\left\{A_{t}: t \in T\right\}$ is *-locally countable at $x_{\lambda} \Rightarrow\left\{\chi_{\left(A_{t}\right)_{(\perp)}}: t \in T\right\}$ is *-locally countable at $x_{\lambda}$.

(ii) $\left\{A_{t}: t \in T\right\}$ is flintily locally countable at $x_{\lambda} \Rightarrow\left\{\chi_{\left(A_{t}\right)_{(\perp)}}: t \in T\right\}$ is flintily locally countable at $x_{\lambda}$.

\subsection{Theorem}

Let $(X, \tau)$ be an $L$-ts, $A \in L^{X}, \mathbf{A}=\left\{A_{t}: t \in T\right\} \subseteq L^{X}$. If $\mathbf{A}$ is flintily locally countable in $A$, then $\mathrm{cl} \mathbf{A}$ is flintily locally countable in $A$.

\subsection{Remark}

Clearly flintily local countability is strictly stronger than *-local countability. But in weakly $\perp$-induced $L$-ts they are coincident with each other.

\subsection{Theorem}

Let $(X, \tau)$ be a weakly $\perp$-induced $L$-ts, $A \in L^{X}, \mathbf{A}=\left\{A_{t}: t \in T\right\} \subseteq$ $L^{X}$. Then $\mathbf{A}$ is flintily locally countable in $A$, if and only if $\mathbf{A}$ is *-locally countable in $A$.

Proof. By Theorem 4.2, it is enough to prove that *-local countability implies flinty local countability. Suppose $\mathbf{A}$ is ${ }^{*}$-local countable in $A$. Let $x_{\lambda} \in M(\downarrow A)$. Then there exist $U \in Q\left(x_{\lambda}\right)$ and a countable subset $T_{0}$ of $T$ such that $t \in T \backslash T_{0} \Rightarrow \chi_{\left(A_{t}\right)_{(\perp)}} \neg q U$ is satisfied. Since $(X, \tau)$ is weakly $\perp$ induced, $U_{(\perp)} \in[\tau]$. Let $t \in T \backslash T_{0}, y \in A_{t(\perp)}$, then $U^{\prime}(y) \geq \chi_{\left(A_{t}\right)_{(\perp)}}(y)=$ $\top$. So $y \in U_{[\top]}^{\prime}=X \backslash U_{(\perp)}$ and hence $\left(\chi_{U_{(\perp)}}\right)^{\prime}(y)=\top=\chi_{\left(A_{t}\right)_{(\perp)}}(y)$. 
That is to say $\chi_{\left(A_{t}\right)_{(\perp)}} \leq\left(\chi_{U_{(\perp)}}\right)^{\prime}, \chi_{\left(A_{t}\right)_{(\perp)}} \neg q\left(\chi_{U_{(\perp)}}\right)$. Since $\chi_{U_{(\perp)}} \in \tau$, $\chi_{U_{(\perp)}} \in Q\left(x_{\lambda}\right) \cap \operatorname{crs}(\tau)$. Hence $\mathbf{A}$ is flintily locally countable.

\subsection{Definition}

Let $(X, \tau)$ be an $L$-ts, $A \in L^{X}, \alpha \in M(L) . A$ is called flintily $\alpha$-paraLindelof if for every open $\alpha$ - $Q$-cover $\Phi$ of $A$, there exist an open refinement $\Psi$ of $\Phi$ which is flintily locally countable in $A$ and $\Psi$ is also an $\alpha-Q$-cover of A. $A$ is called flintily para-Lindelof if $A$ is flintily $\alpha$-para-Lindelof for every $\alpha \in M(L)$. And $(X, \tau)$ is flintily para-Lindelof if $T$ is flintily para-Lindelof.

By Theorem 4.2, the following implications hold:

\subsection{Theorem}

Let $(X, \tau)$ be an $L$-ts, $A \in L^{X}, \alpha \in M(L)$, then

(i) $A$ is flintily $\alpha$-para-Lindelof $\Rightarrow A$ is $\alpha^{*}$-para-Lindelof $\Rightarrow$ is $\alpha$-paraLindelof.

(ii) $A$ is flintily para-Lindelof $\Rightarrow A$ is ${ }^{*}$-para-Lindelof $\Rightarrow A$ is para-Lindelof.

Similar to Theorem 3.5 we can prove that flintily para-Lindelofness is hereditary with respect to closed subsets.

\subsection{Theorem}

Let $(X, \tau)$ be an $L$-ts, $A \in L^{X}, \alpha \in M(L), B \in \tau^{\prime}$. Then

(i) $A$ is flintily $\alpha$-para-Lindelof $\Rightarrow A \wedge B$ is flintily $\alpha$-para-Lindelof.

(ii) $A$ is flintily para-Lindelof $\Rightarrow A \wedge B$ is flintily para-Lindelof.

\subsection{Theorem}

In a weakly induced $L$-ts $(X, \tau)$, the following are equivalent

(i) $(X, \tau)$ is flintily para-Lindelof.

(ii) There exist $\alpha \in M(L)$ such that $(X, \tau)$ is flintily $\alpha$-para-Lindelof;

(iii) $(X,[\tau])$ is para-Lindelof.

Proof. $\quad$ (i) $\Rightarrow$ (ii) is obvious.

(ii) $\Rightarrow$ (iii): Let $\mathbf{U}$ be an open cover of $(X,[\tau])$. Then $\Phi=\left\{\chi_{U}: U \in \mathbf{U}\right\}$ is an open $\alpha$ - $Q$-cover of $T$ and by (ii) it has an open and flintily locally countable refinement $\Psi=\left\{A_{t}: t \in T\right\}$ such that $\Psi$ is an $\alpha$-Q-cover of $\top$. For every $t \in T$, take $V_{t}=A_{t\left(\alpha^{\prime}\right)}$ and $\mathbf{V}=\left\{V_{t}: t \in T\right\}$. Then by the weakly induced property of $(X, \tau), \mathbf{V}$ is an open cover of $(X,[\tau])$. Now we will prove $\mathbf{V}$ is a locally countable refinement of $\mathbf{U}$. Let $V_{t} \in \mathbf{V}$. Since 
is $\Psi$ a refinement of $\Phi$, there exist $U \in \mathbf{U}$ such that $A_{t} \leq \chi_{U}$. Suppose $x \in V_{t}$, then $A_{t}(x) \not \leq \alpha^{\prime}$, so $\chi_{U}(x) \neq \perp, x \in U, V_{t} \subset U$. Therefore $\mathbf{V}$ is a refinement of $\mathbf{U}$.

Let $x \in X$. Since $\Psi$ is flintily locally countable, there exist $B \in Q\left(x_{\alpha}\right) \cap$ $\operatorname{crs}(\tau)$ such that $A_{t} q B$ for only a countable number of members $A_{t}$ s in $\psi$. Since $B \in Q\left(x_{\alpha}\right)$ is crisp, $B_{(\perp)}$ is the neighbourhood of $x$. For every $t \in T$ if $A_{t} \neg q B$, then $V_{t} \cap B_{(\perp)}=\Phi$. So $B_{(\perp)}$ intersects with only a countable members of $\mathbf{V}$, thus $\mathbf{V}$ is locally countable. Hence $(X,[\tau])$ is paraLindelof. (iii) $\Rightarrow$ (i) suppose $\alpha \in M(L), \mathbf{A}=\left\{A_{t}: t \in T\right\}$ is an open $\alpha$-Q-cover of $\top$. For every $t \in T$ take $U_{t}=A_{t\left(\alpha^{\prime}\right)}$ and $\mathbf{U}=\left\{U_{t}: t \in T\right\}$. Since $\mathbf{A}$ is an open $\alpha$-Q-cover of $\top$ and $(X, \tau)$ is weakly induced, $\mathbf{U}$ is an open cover of $(X,[\tau])$. Therefore by (iii), there exists an open and locally countable refinement $\mathbf{V}=\left\{V_{s}: s \in S\right\}$ of $\mathbf{U}$ which is also a cover of $(X,[\tau])$. For every $s \in S$ take $t(s) \in T$ such that $V_{s} \subset U_{t(s)}$, let $W_{s}=A_{t(s)} \wedge \chi \gamma_{s}$ then $W s$ is an open $L$-set and $W s \leq A_{t(s)}$ for every $s \in S$. Therefore $\mathbf{W}=\{W s: s \in S\}$ is an open refinement of $\mathbf{A}$. Now we will show that $\mathbf{W}$ is an open $\alpha$-Q-cover of $\top$. Let $x_{\alpha} \in M\left(L^{X}\right)$ take $s \in S$ such that $x \in V_{s}$ and hence $x \in U_{t(s)}$. So $A_{t(s)}(x) \not \leq \alpha^{\prime}, \alpha \not \leq A_{t(s)}(x)^{\prime}$. Since $x \in V_{s}$, $\chi_{V s} \in Q\left(x_{\alpha}\right)$, we have $W s=A_{t(s)} \wedge \chi_{V s} \in Q\left(x_{\alpha}\right)$. Hence $\mathbf{W}$ is an open $\alpha$ - $Q$-cover of $\top$.

Suppose $x_{\alpha} \in M\left(L^{X}\right)$, then since $\mathbf{V}$ being locally countable in $(X,[\tau])$, there exist a neighbourhood $B$ of $x$ in $(X,[\tau])$ such that $B$ intersects with only countably many members of $\mathbf{V}$ say $V_{s_{0}}, V_{s_{1}}, V_{s_{2}}, \cdots$. Then for every $s \in S \backslash\left\{s_{0}, s_{1}, s_{2} \ldots\right\}, V s \cap B=\Phi, B \subset V s^{\prime}$ and thus $\chi_{B} \leq \chi_{V_{s}^{\prime}} \leq$ $A_{t(s)}^{\prime} \vee \chi_{V_{s}}^{\prime}=W s^{\prime}$. That is $\chi_{B} \neg q W s$. Hence $\mathbf{W}$ is flintily locally countable. This completes the proof.

\section{Invariant Theorems}

In this section we study the behaviour of para-Lindelof spaces under various types of fuzzy mappings.

\subsection{Definition}

[17] Let $(X, \tau),(Y, \mu)$ be $L$-topological spaces, $f: X \rightarrow Y$ be an ordinary mapping. Based on this we define the $L$-fuzzy mapping $f^{\rightarrow}: L^{X} \rightarrow L^{Y}$ and its $L$-fuzzy reverse mapping $f^{\leftarrow}: L^{Y} \rightarrow L^{X}$ by $f^{\rightarrow}: L^{X} \rightarrow L^{Y}, f^{\rightarrow}(A)(y)=\vee\{A(x): x \in X, f(x)=y\} \forall A \in L^{X}, \forall y \in Y$. $f^{\leftarrow:} L^{Y} \rightarrow L^{X}, f^{\leftarrow}(B)(x)=B(f(x)), \forall B \in L^{Y}, \forall x \in X$. 


\subsection{Definition}

[17] Let $(X, \tau),(Y, \mu)$ be $L$-topological spaces,

$f \rightarrow: L^{X} \rightarrow L^{Y}$ an $L$-fuzzy mapping. We say $f \rightarrow$ is an $L$-fuzzy continuous mapping from $(X, \tau)$ to $(Y, \mu)$ if its $L$-fuzzy reverse mapping $f \leftarrow: L^{Y} \rightarrow L^{X}$ maps every open subset in $(Y, \mu)$ as an open one in $(X, \tau)$. i.e., $\forall V \in \mu$, $f^{\leftarrow}(V) \in \tau$.

\subsection{Definition}

[17] Let $(X, \tau),(Y, \mu)$ be $L$-topological spaces, $f^{\rightarrow}: L^{X} \rightarrow L^{Y}$ an $L$-fuzzy mapping. We say $f^{\rightarrow}$ is open if it maps every open subset in $(X, \tau)$ as an open one in $(Y, \mu)$. i.e., $\forall U \in \tau, f \rightarrow(U) \in \mu$.

\subsection{Definition}

[17] Let $(X, \tau),(Y, \mu)$ be $L$-topological spaces, $f^{\rightarrow}: L^{X} \rightarrow L^{Y}$ an $L$-fuzzy mapping. We say $f^{\rightarrow}$ is closed if it maps every closed subset in $(X, \tau)$ as an closed one in $(Y, \mu)$. i.e., $\forall F \in \tau^{\prime}, f^{\rightarrow}(F) \in \mu^{\prime}$.

\subsection{Definition}

[1] Let $(X, \tau),(Y, \mu)$ be $L$-ts's, $f \rightarrow: L^{X} \rightarrow L^{Y}$ an $L$-fuzzy mapping. Then $f \rightarrow$ is perfect if it is continuous, closed and $f^{\leftarrow}(y)$ is $N$-compact for every $y \in Y$.

\subsection{Result}

[17] If $(X, \tau),(Y, \mu)$ are two weakly induced $L$-topological spaces, then

(i) If the map $f^{\rightarrow}: L^{X} \rightarrow L^{Y}$ is $L$-fuzzy continuous, then $f:(X,[\tau]) \rightarrow$ $(Y,[\mu])$ is continuous;

(ii) If the map $f^{\rightarrow}: L^{X} \rightarrow L^{Y}$ is $L$-fuzzy closed, then $f:(X,[\tau]) \rightarrow(Y,[\mu])$ is closed;

(iii) If the map $f^{\rightarrow}: L^{X} \rightarrow L^{Y}$ is $L$-fuzzy open, then $f:(X,[\tau]) \rightarrow(Y,[\mu])$ is open.

\subsection{Theorem}

Let $(X, \tau),(Y, \mu)$ are two weakly induced $L$-topological spaces. Then if $f \rightarrow: L^{X} \rightarrow L^{Y}$ is perfect, then so is $f:(X,[\tau]) \rightarrow(Y,[\mu])$. 
Proof. Let $y_{\alpha} \in M\left(L^{Y}\right)$. Since $f \rightarrow: L^{X} \rightarrow L^{Y}$ is perfect, $f \leftarrow\left(y_{\alpha}\right)$ is $N$-compact. Now to prove $f:(X,[\tau]) \rightarrow(Y,[\mu])$ is perfect, it is enough to prove that $f^{\leftarrow}\left(y_{\alpha}\right)$ is compact for every $y \in Y$. Now let $\mathbf{U} \in[\tau]$ be an open cover of $f^{-1}(y)$. Consider $\mathbf{U} *=\left\{\chi_{U}: U \in \mathbf{U}\right\}$. This is an open $\alpha$ - $Q$-cover of $f^{\leftarrow}\left(y_{\alpha}\right)$. For, let $x_{\alpha} \leq f^{\leftarrow}\left(y_{\alpha}\right)$. i.e., $f^{\leftarrow}\left(y_{\alpha}\right)(x)=y_{\alpha}(f(x)) \geq \alpha$. Now let $U \in \mathbf{U}$ be such that $x \in U$. This is possible since $U$ is a cover of $f^{-1}(y)$. Then $\chi_{U}(x) \geq y_{\alpha} \geq \alpha$. i.e., $\chi_{U}(x) \geq \alpha$ or $x_{\alpha} \leq \chi_{U}$. Hence clearly $x_{\alpha} q \chi_{U}$. Hence $\left\{\chi_{U}: U \in \mathbf{U}\right\}$ is an open $\alpha$-Q-cover of $f^{\leftarrow}\left(y_{\alpha}\right)$.

Again $f^{\leftarrow}\left(y_{\alpha}\right)$ being $N$-compact, there exists a finite sub collection $\mathbf{U}_{s}^{*}$ of $\mathbf{U}^{*}$ which is also an $\alpha^{-}-Q$ cover of $f^{\leftarrow}\left(y_{\alpha}\right)$. Let $\mathbf{U}_{s}^{*}=\left\{\chi_{U 1}, \chi_{U 2}, \cdots, \chi_{U k}\right\}$. Then clearly $\left\{U_{1}, U_{2}, \cdots, U_{k}\right\}$ will be a finite sub cover of $f^{-1}(y)$. This completes the proof.

\subsection{Theorem}

$(X, \tau),(Y, \mu)$ are two weakly induced $L$-tss. If $(X, \tau)$ is para-Lindelof and $f^{\rightarrow}: L^{X} \rightarrow L^{Y}$ be a closed map with $f^{\leftarrow}\left(y_{\alpha}\right)$ Lindelof for each $y_{\alpha} \in M\left(L^{Y}\right)$, then $(Y, \mu)$ is para-Lindelof.

Proof. Let $\mathbf{U}$ be an open $\alpha$-Q-cover of $Y$ and let $\mathbf{W}=\left\{W_{t}: t \in T\right\}$ be a locally countable open $\alpha$-Q-cover refinement of $\left\{f^{\leftarrow}(U): U \in \mathbf{U}\right\}$. Now for any $y_{\alpha} \in M\left(L^{Y}\right), f^{\leftarrow}\left(y_{\alpha}\right)$ is Lindelof so there is an open set $G_{y \alpha}$ in $L^{X}$ such that $f^{\leftarrow}\left(y_{\alpha}\right) \leq G_{y \alpha}$ and $G_{y \alpha} \leq W_{t}$ for countably many $t \in T$. Take $V_{y \alpha}$ as the saturated part of $G_{y \alpha}$. Then $f^{\rightarrow}\left(V_{y \alpha}\right)$ is an open set about $y_{\alpha}$. Consider $\mathbf{H}=\left\{f^{\rightarrow}\left(W_{t}\right): W_{t} \in \mathbf{W}\right\}$. Now $f^{\rightarrow}\left(V_{y \alpha}\right)$ meeting only countably many elements of $\mathbf{H}$. Hence $\mathbf{H}$ is locally countable and it is clear that $y_{\alpha} \in \operatorname{int}\left(\operatorname{st}\left(y_{\alpha}, \mathbf{H}\right)\right)$ for every $y_{\alpha} \in L^{Y}$. Since $\mathbf{H}$ is a refinement of $\mathbf{U}$, it follows from Theorem 3.9 that $(Y, \mu)$ is para-Lindelof.

Now by Theorem 5.7 we readily have

\subsection{Theorem}

$(X, \tau),(Y, \mu)$ are two weakly induced $L$-tss and $f^{\rightarrow}: L^{X} \rightarrow L^{Y}$ be a perfect map. Then $(X, \mu)$ is para-Lindelof if and only if $(Y, \mu)$ is para-Lindelof.

A similar result we can obtain for flintily para-Lindelof space also:

\subsection{Theorem}

$(X, \tau),(Y, \mu)$ are two weakly induced $L$-tss and $f^{\rightarrow}: L^{X} \rightarrow L^{Y}$ be a perfect map. Then $(X, \mu)$ is flintily para-Lindelof if and only if $(Y, \mu)$ is 
flintily para-Lindelof.

\section{References}

[1] Baiju, T. and Sunil Jacob John, Finitistic Spaces in L-topological spaces, Proyecciones Journal of Mathematics 28 (1), pp. 47-56, (2009).

[2] Baiju, T. and Sunil Jacob John, Fuzzy submetacompact spaces. (communicated)

[3] Baiju, T. and Sunil Jacob John, Subparacompactness in $L$-topological spaces (communicated).

[4] Burke, D. K, Paralindelof spaces and closed mappings, Topology Proc. 5, pp. 47-57, (1980).

[5] Burke, D. K, Refinements of locally countable collections, Topology Proc. 4, pp. 19-27, (1979).

[6] Chang, C. L. Fuzzy Topological Spaces, J. Math. Anal. Appl. 24, pp. 182-190, (1968).

[7] Dieudonne, J. Une generalization des espaces compact, J. Math. Pures. Appl. 23, pp. 65-76, (1944).

[8] Fleissner, W. G, and Reed, G. M., Para-Lindelof spaces and spaces with a $\sigma$-locally countable base, Topology Proc. 2, pp. 89-110, (1977).

[9] Greever, J. On some generalized compactness properties, Publ. Res. Inst. Math. Soc., Ser. A 4 (1), 39-49, (1968).

[10] Hohle, U. and Rodabaugh, S. E. Mathematics of Fuzzy Sets : Logic, Topology and Measure Theory, The Hand Book of Fuzzy Set Series 3, Kluwer Academic Pub. (1999).

[11] Kubiak, T. The topological modification of the L-fuzzy unit interval, in: S.E. Rodabaugh, E.P. Klement, U. Hohle (Eds.), Applications of Category Theory to Fuzzy Subsets, (Kluwer Academic Publishers, Dordrecht, pp. 275-305, (1992).

[12] Lowen, R., Fuzzy Topological Spaces and Fuzzy Compactness, J. Math. Anal. Appl. 56, pp. 621-633, (1976). 
[13] Luo Mao-Kang, Paracompactness in fuzzy topological spaces, J. Math. Anal. Appl. 130 (1), pp. 55-77, (1988).

[14] Sunil Jacob John and Baiju, T. Metacompactness in $L$-topological spaces, Iranian Journal of Fuzzy Systems 5 (3), pp. 71-79, (2008).

[15] Wang, G. J. On the structure of fuzzy lattices, Acta math. Sinica 29, pp. 539-543, (1986).

[16] Wang, G. J. Theory of L-fuzzy topological spaces, Shaanxi Normal University Pub., Xian (1988).

[17] Ying - Ming Liu and Mao-Kang Luo, Fuzzy Topology, Advances in Fuzzy SystemsApplications and Theory Vol. 9, World Scientific, (1997).

[18] Zadeh, L. A. Fuzzy sets, Information and Control 8, pp. 338-353, (1965).

\section{T. Baiju}

Department of Mathematics

National Institute of Technology Calicut

Calicut 673601

Kerala

India

e-mail : baijutmaths@gmail.com

and

\section{Sunil Jacob John}

Department of Mathematics

National Institute of Technology Calicut

Calicut 673601

Kerala

India

e-mail : sunil@nitc.ac.in 